\title{
MULTISCALE HOMOGENIZATION MODELING OF ALKALI-SILICA-REACTION DAMAGE IN CONCRETE
}

\author{
R. REZAKHANI*, M. ALNAGGAR ${ }^{\dagger}$ AND G. CUSATIS* \\ *Northwestern University \\ Evanston, IL USA \\ e-mail: rrezakhani@u.northwestern.edu - g-cusatis@ northwestern.edu \\ ${ }^{\dagger}$ Rensselaer Polytechnic Institute \\ Troy, NY USA \\ e-mail: alnagm2@rpi.edu
}

Key words: Multiscale Homogenization, Asymptotic Expansion, Alkali-Silica-Reaction

\begin{abstract}
Alkali silica reaction (ASR) is one of the main reasons that cause deterioration in concrete structures, such as dams and bridges. ASR is a chemical reaction between alkali ions from cement paste and the silica inside each aggregate piece. ASR gel, which is the product of this reaction, imbibes additional water causing swelling and cracking, which leads to degradation of concrete mechanical properties. In this study, to model ASR-induced damage, the Lattice Discrete Particle Model (LDPM) is adopted, which is a meso-scale discrete model. LDPM simulates concrete at the level of coarse aggregate pieces. ASR effects have been already successfully modeled by LDPM in the recent past. This paper employs a recently developed multiscale homogenization approach to derive macroscopic constitutive equations for ASR-damaged concrete. The adopted homogenized model is used to reproduce experimental data on volumetric expansion of unrestrained concrete prisms.
\end{abstract}

\section{Introduction}

Alkali-Silica-Reaction (ASR) in concrete is a chemical reaction between the silica existing in aggregate pieces and alkali ions contained in the cement paste. In the presence of water, ASR produces an expansive gel, regularly called "ASR gel", which cause cracking and damage in concrete over time. As a result of this chronic material deterioration, both strength and stiffness of concrete are decreased. Since presence of water is the cornerstone in ASR occurrence, it is reported in concrete structures exposed to high humidity environments, usually above $60 \%$. In addition, temperature also plays a crucial role in driving ASR effects.

Stanton [1] studied the ASR effect for the first time with reference to various aspects in- cluding: chemistry of the reaction, structural and material consequences, development of experimental techniques to assess the vulnerability of aggregate and mixes to ASR, and the formulation of new strategies to mitigate the ASR effects. Since then, several research efforts have been devoted to scrutinize different aspects of ASR.

In general, experiments on ASR effect have been performed on small scale accelerated ASR laboratory specimens. Among multiple experimental techniques is the Accelerated Mortar Bar Test (AMBT) which is completed within 16 days. AMBT experiments are performed on specimens, made of crushed aggregate pieces, which are immersed in an alkali rich solution, while temperature is raised to $80^{\circ} \mathrm{C}$. Concrete Prism Test (CPT) is another testing method 
which provides broader insight into the ASR reaction on real structures. CPTs are usually carried out on specimens that are made of aggregates used in practice and are totally sealed to maintain the relative humidity at high values.

As far as numerical modeling of ASR effects are concerned, numerous research efforts have been carried out to explore this phenomenon at different length scales and with various accuracy levels. Bažant [2] was the first to study ASR deterioration by means of fracture mechanics approach and to predict the pessimum aggregate size.

Multiple macroscopic models are available in the literature to investigate ASR mechanisms and related degradation of mechanical properties. The phenomenological model developed by Charlwood et al. [3] is one of the earliest ones. Later, researchers formulated computational models based on the ASR kinetics including more details on creep effect as well as cracking and implemented them into the finite element framework using the crack band approach [4]. These models allowed successfully replicating some experimantal data on ASR expansion [5]. Effect of stress state on ASR deterioration mechanism were incoporated into this type of models by Saouma and Perotti [6]. Comi et al. [7] proposed damage models which included chemical and mechanical aspects of the ASR process in a consistent thermodynamic fashion. Humidity and temperature effects were integrated into the ASR reaction kinetics law by Poyet et al. [8]. While all aformentioned models were deterministic, Capra and Sellier [9] studied ASR phenomena by a probabilistic approach. Detailed consideration of the chemical aspect of ASR reaction were addressed by Saouma et al. [10] who derived an analytical model to explain the kinetics of the alkali-silica chemical reaction.

The main drawback of all the aforementioned models is their inability to simulate accurately crack patterns induced by ASR reaction, which hinders the prediction of ASR degradation effects and requires the assumption of phenomenological relationships between concrete strength and ASR gel expansion. In addition, it limits the ability of such models to explain complex triaxial behavior of concrete under ASR and also forces the assumption of phenomenological relationships between ASR gel expansion and stress state. These limitations are inherently related to simulating concrete as an isotropic and homogenous continuum [11, 12]. The ASR-LDPM model [13, 14] used in the present study is the first model to overcome all aforementioned limitations. The model integrates ASR effects within the Lattice Discrete Particle Model (LDPM) [15, 16]. LDPM simulates the mechanical interaction of coarse aggregate pieces through a system of threedimensional polyhedral particles, each resembling a spherical coarse aggregate piece with its surrounding mortar, connected through lattice struts. LDPM has the ability of simulating the effect of material heterogeneity on the fracture processes. ASR-LDPM can reproduce realistic crack patterns under free expansion, restrained expansion and expansion under various loading conditions. In addition, it can replicate the deterioration of concrete strength and stiffness, temperature effects on reaction kinetics and alkali content variations. It is worth noting that, ASRLDPM can predict degradation of mechanical properties without the need of any phenomenological relationship between such degradation and expansion.

Despite of all above mentioned capabilities, major limitation of employing ASR-LDPM in the simulation of large concrete structural members is the immense computational cost. This is due to the fact that LDPM explicitly simulates concrete aggregate pieces that are each represented as a computational node with six degrees of freedom. In addition, each computational node is surrounded by many triangular facets, forming polyhedral cells, which are the computational entities over which constitutive calculations are carried out during each computational step. As a result, it is necessary to establish a multiscale framework to increase computational efficiency [17]. Wu et al. [18] established a FE2 homogenization framework 
based on averaging theorem to analyze ASR effect in concrete. A multiscale homogenization framework has been recently developed by the authors [11, 19,-21] to homogenize discrete models to continuum, and this homogenization scheme has been employed to evaluate elastic effective properties of concrete as well as its nonlinear response under different loading conditions.

\section{ASR theory and modeling technique}

ASR fundamental governing equations along with the computational framework for modeling ASR effect in concrete is discussed in this section.

\subsection{ASR governing equations}

In the current paper, the ASR model developed in [13] is considered and incorporated in the LDPM framework, which is employed as the meso-scale model in the homogenization scheme. The theory is established for the meso-scale, scale of coarse aggregate pieces, and lower scale mechanisms are taken into account in an average sense by means of the governing constitutive equations.

Gel formation. To form the ASR gel, water and alkali ions must reach the silica present in the aggregate pieces through a diffusion process through the aggregate volume. Therefore, gel mass $M_{g}$ produced from an aggregate piece of diameter $D$ is obtained from the solution of a steady state mass balance of radial diffusion process into the aggregate piece. Figure 1 a shows the diffusion process that takes place during ASR reaction, in which $z$ is the diffusion front position measured from the aggregate center or the radius of the aggregate unreacted portion. Thus, one can write,

$$
M_{g}=\kappa_{a} \kappa_{g} \frac{\pi}{6}\left(D^{3}-8 z^{3}\right)
$$

where $\kappa_{a}=\min \left(\left\langle c_{a}-c_{a 0}\right\rangle /\left(c_{a 1}-c_{a 0}\right), 1\right)$ is considered to account for the fact that the available alkali content in the cement paste around the vicinity of each aggregate piece is not necessarily enough for a complete ASR reaction. $c_{a 0}$ is the threshold alkali content at which, no or minimal expansion is observed, and $c_{a 1}$ is the saturation alkali content enough for the ASR reaction to occur completely. $\kappa_{g}$ is a free parameter. Rate of the diffusion front position can be written as

$$
\dot{z}=-\kappa_{z} \frac{w_{e} \exp \left(\frac{E_{a g}}{R T_{0}}-\frac{E_{a g}}{R T}\right)}{z\left(1-\frac{2 z}{D}\right)}
$$

where $T_{0}=$ reference temperature; $T=$ current temperature; $E_{a g}=$ activation energy of the diffusion process; and $R=$ universal gas constant. $w_{e}=$ density of water content in concrete surrounding the aggregate piece estimated as $w_{e}=$ $\left(w / c-0.188 \alpha_{c}^{\infty}\right) c$ at saturation; in which $\alpha_{c}^{\infty}=$ $(1.031 w / c) /(0.194+w / c)$ asymptotic hydration degree, $w / c=$ water-to-cement ratio, $c=$ cement content, and $\kappa_{z}$ is a free parameters.

Water imbibition. The gel created through ASR reaction has the capacity to imbibe water molecules and expand subsequently. This exerts an expansive pressure on the surrounding concrete and result in cracking and damage. The water imbibition process can be formulated by relating the rate of the imbibed water mass by ASR gel $M_{i}$ to the thermodynamic driving force and a characteristic imbibition time. Considering the developed equation for the gel mass $M_{g}$ given by the integration of Eq. 2, the rate of imbibed water reads

$\dot{M}_{i}=\frac{C_{i}^{1}}{\delta^{2}} \exp \left(-\eta M_{i}\right)\left[\kappa_{i} M_{g}-M_{i}\right] \exp \left(\frac{E_{a i}}{R T_{0}}-\frac{E_{a i}}{R T}\right)$

in which, at thermodynamic equilibrium, a linear relationship is considered between the rate of water imbibition and the mass of formed gel through $\kappa_{i}$ as the constant of proportionality. $C_{i}^{1}$ is the initial micro-diffusivity of the of water close to aggregate surface. The exponentially decaying factor in Eq. 3 represents the reduction of water imbibition rate as more water is imbibed into ASR gel. This is a reasonable relation, since diffusivity of ASR gel decreases during the water imbibition process. In 


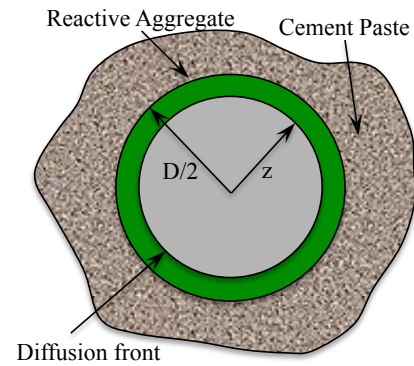

(a)

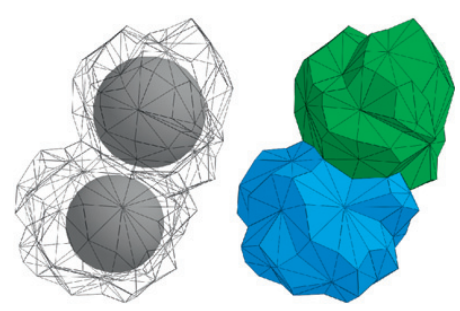

(b)

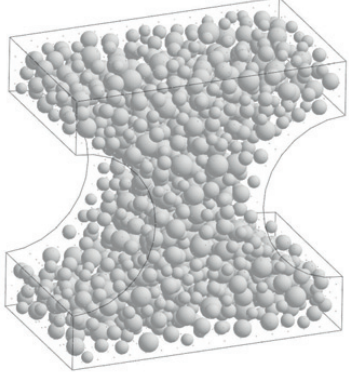

(c)

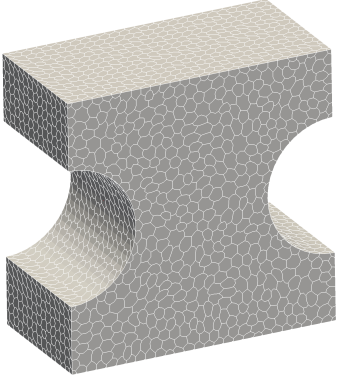

(d)

Figure 1: (a) ASR gel created around a generic aggregate piece. (b) LDPM polyhedral cell enclosing spherical aggregate pieces. (c) Spherical particles and (d) polyhedral cell representations of a typical dogbone specimen.

addition, the temperature-dependence is formulated through an Arrhenius-type equation governed by the activation energy $E_{a i}$ of the imbibition process. $\delta$ is the average (or effective) distance of water transport process from the concrete around the aggregate into the ASR gel.

\subsection{The Lattice Discrete Particle Model (LDPM)}

The Lattice Discrete Particle Model (LDPM) [15, 16] is a meso-scale discrete model that simulates the mechanical interaction of coarse aggregate pieces embedded in a cementitious matrix (mortar). The geometrical representation of concrete mesostructure is constructed through the following steps. 1) The coarse aggregate pieces, whose shapes are assumed to be spherical, are introduced into the concrete volume by a try-and-reject random procedure. 2) Zeroradius aggregate pieces (nodes) are randomly distributed over the external surfaces to facilitate the application of boundary conditions. 3) A three-dimensional domain tessellation, based on the Delaunay tetrahedralization of the generated aggregate centers, creates a system of polyhedral cells (see Figure $1 \mathrm{~b}$ ) interacting through triangular facets and a lattice system composed by the line segments connecting the particle centers. Figures $1 \mathrm{k}$ and $\mathrm{d}$ represent spherical particle and corresponding polyhedral representations of a typical dogbone specimen.

In LDPM, rigid body kinematics is used to describe the deformation of the lattice/particle system and the displacement jump, $\llbracket \mathbf{u}_{C} \rrbracket$, at the centroid of each facet is used to define measures of strain as

$\epsilon_{N}=\frac{\mathbf{n}^{\mathrm{T}} \llbracket \mathbf{u}_{C} \rrbracket}{\ell} ; \quad \epsilon_{L}=\frac{\mathbf{l}^{\mathrm{T}} \llbracket \mathbf{u}_{C} \rrbracket}{\ell} ; \quad \epsilon_{M}=\frac{\mathbf{m}^{\mathrm{T}} \llbracket \mathbf{u}_{C} \rrbracket}{\ell}$

where $\ell=$ interparticle distance; and $\mathbf{n}, \mathbf{l}$, and $\mathbf{m}$, are unit vectors defining a local system of reference attached to each facet.

Next, a vectorial constitutive law governing the behavior of the material is imposed at the centroid of each facet. In the elastic regime, the normal and shear stresses are proportional to the corresponding strains: $t_{N}=E_{N} \epsilon_{N}^{*}=$ $E_{N}\left(\epsilon_{N}-\epsilon_{N}^{\xi}\right) ; t_{M}=E_{T} \epsilon_{M}^{*}=E_{T}\left(\epsilon_{M}-\epsilon_{M}^{\xi}\right) ; t_{L}=$ $E_{T} \epsilon_{L}^{*}=E_{T}\left(\epsilon_{L}-\epsilon_{L}^{\xi}\right)$, where $E_{N}=E_{0}, E_{T}=\alpha E_{0}$, $E_{0}=$ effective normal modulus, and $\alpha=$ shearnormal coupling parameter; and $\epsilon_{N}^{\xi}, \epsilon_{M}^{\xi}, \epsilon_{L}^{\xi}$ are mesoscale eigenstrains that might arise from a variety of phenomena such as, but not limited to, thermal expansion, shrinkage, and ASR expansion.

For stresses and strains beyond the elastic limit, LDPM mesoscale nonlinear phenomena are characterized by three mechanisms: 1) Fracture and cohesion due to tension and tension-shear, 2) Compaction and pore collapse from compression, and 3) Friction due to compression-shear. One can find the details of the corresponding constitutive equations in [15]. Finally, the governing equations of the 
LDPM framework are completed through the equilibrium equations of each individual particle.

\subsection{Modeling ASR effect within LDPM framework}

The rate of imbibed water mass $\dot{M}_{i}$ by a generic aggregate piece of diameter $D$ is presented in Eq. 3. In this equation, the radius of a generic expanded aggregate piece of initial radius of $r=D / 2$ can be written as

$$
r_{i}=\left(3 M_{i} / 4 \pi \rho_{w}+r^{3}\right)^{1 / 3}
$$

where $\rho_{w}$ is the mass density of water. Using the chain rule along with Equation 5, the rate of aggregate radius increase can be calculated as

$$
\begin{aligned}
\dot{r}_{i} & =\frac{d r_{i}}{d t}=\frac{d r_{i}}{d M_{i}} \frac{d M_{i}}{d t}=\dot{M}_{i} \frac{d r_{i}}{d M_{i}} \\
& =\frac{\dot{M}_{i}}{4 \pi \rho_{w}}\left(3 M_{i} / 4 \pi \rho_{w}+r^{3}\right)^{-2 / 3}
\end{aligned}
$$

Therefore, for a generic facet in LDPM geometry, the incompatible eigenstrain rate due to ASR gel expansion $\dot{\epsilon}_{N}^{a s r}$ is determined as follows

$$
\dot{\epsilon}_{N}^{a s r}= \begin{cases}0.0 & \text { if } z=0 \\ \left(\dot{r}_{i 1}+\dot{r}_{i 2}\right) / \ell & \text { if } z>0\end{cases}
$$

where $\dot{r}_{i 1}$ and $\dot{r}_{i 2}$ are the radius increase of the two aggregate pieces which share the considered facet. It should be noted that it is herein assumed that the shear eigenstrains due to gel expansion imposed on each facet are approximately negligible, $\epsilon_{M}^{a s r}=\epsilon_{L}^{a s r} \approx 0$, although this may not be an exact assumption due to the irregular shape of actual aggregate pieces.

The presented formulation, is implemented into MARS, a multi-purpose computational code for the explicit dynamic simulation of structural performance [22].

\section{Multiscale homogenization theory}

In this section, fundamentals and crucial aspects of a general multiple scale homogenization framework proposed by Rezakhani and
Cusatis [19] based on classical asymptotic analysis is discussed. In the development of the homogenization theoretical framework, a particulate material structure resembeling LDPM is considered as the underlying material domain, see Figure 3 .

\subsection{Fine-scale problem definition}

Figure 3 a shows the interaction of two neighboring particles, $I$ and $J$, which share a generic facet. For the case of small strains and displacements, facet strains, already defined in Equation 4 , and curvatures can be written in terms of particles displacement and rotation vectors

$$
\begin{gathered}
\epsilon_{\alpha}^{I J}=\frac{1}{r}\left(\mathbf{U}^{J}+\boldsymbol{\Theta}^{J} \times \mathbf{c}^{J}-\mathbf{U}^{I}-\boldsymbol{\Theta}^{I} \times \mathbf{c}^{I}\right) \cdot \mathbf{e}_{\alpha}^{I J} \\
\chi_{\alpha}^{I J}=\frac{1}{r}\left(\boldsymbol{\Theta}^{J}-\boldsymbol{\Theta}^{I}\right) \cdot \mathbf{e}_{\alpha}^{I J}
\end{gathered}
$$

where $\alpha=N, M, L ; r=\left|\mathbf{x}^{I J}\right| ; \chi_{\alpha}^{I J}=$ facet curvatures; $\mathbf{x}^{I J}=\mathbf{x}^{J}-\mathbf{x}^{I}$ is the vector connecting the particle nodes $P_{I}$ and $P_{J} ; \mathbf{e}_{\alpha}^{I J}$ are unit vectors defining a facet Cartesian system of reference such that $\mathbf{e}_{N}^{I J}=$ is orthogonal to the facet and $\mathbf{e}_{N}^{I J} \cdot \mathbf{x}^{I J}>0 ; \mathbf{U}^{I}, \mathbf{U}^{J}=$ displacement vectors of node $P_{I}$ and $P_{J} ; \boldsymbol{\Theta}^{I}, \boldsymbol{\Theta}^{J}=$ rotation vectors of node $P_{I}$ and $P_{J}$; and $\mathbf{c}^{I}, \mathbf{c}^{J}=$ vectors connecting nodes $P_{I}$ and $P_{J}$ to the facet centroid, see Fig. 3a. It must be noted here that displacements and rotations are assumed to be independent variables.

Stress traction $\mathbf{t}^{I J}$ on each facet for a given strain vector is derived through a vectorial constitutive equation. In general, it can be written that $\mathbf{t}^{I J}=t_{\alpha}\left(\epsilon_{N}, \ldots\right) \mathbf{e}_{\alpha}^{I J}$ where summation rule applies over $\alpha$. For instance, in the case of elastic behavior, constitutive equation can be formulated through; $t_{\alpha}^{I J}=E_{\alpha} \epsilon_{\alpha}^{I J}$; in which each traction component and the associated strain are related through $E_{\alpha}$ which is fine-scale elastic constant (summation rule does not apply). Nonlinear facet constitutive equations are explained in detail in [15], with reference to the LDPM which is considered in the numerical examples.

The final step in constituting the discrete computational model is imposing the equilibrium of each single particle under the effect of 
all neighboring particles. Translational and rotational dynamic equilibrium equations of a single particle reads

$$
\begin{gathered}
\mathbf{M}_{u}^{I} \ddot{\mathbf{U}}^{I}-V^{I} \mathbf{b}^{0}=\sum_{\mathcal{F}_{I}} A \mathbf{t}^{I J} \\
\mathbf{M}_{\theta}^{I} \ddot{\boldsymbol{\Theta}}^{I}=\sum_{\mathcal{F}_{I}} A \mathbf{w}^{I J}
\end{gathered}
$$

where $\mathbf{w}^{I J}=\mathbf{c}^{I} \times \mathbf{t}^{I J}$ is the moment of the traction $\mathbf{t}^{I J}$ with respect to the particle node $P_{I} ; \mathcal{F}_{I}$ is the set of facets enclosing node $\mathrm{P}_{I}$, which is obtained by collecting all the facets associated with each node pair $(I, J)$ in which $J$ accounts for all particles that are in contact with particle $I ; A=$ facet area; superimposed dots represent time derivatives; $V^{I}$ is the particle volume; $\mathbf{b}^{0}$ is the body force vector; $\mathbf{M}_{u}^{I}=$ mass tensor of the node $P_{I} ; \mathbf{M}_{\theta}^{I}=$ moment of inertia tensor.

\subsection{Asymptotic expansion homogenization}

Figure $3 \mathrm{p}$ illustrates a generic macroscopic material domain in a global coordinate system $\mathbf{X}$. Two distinct length scales and the related local coordinate systems, $\mathbf{x}$ and $\mathbf{y}$, are introduced at any point of the material domain to represent the local macro- and meso-scale problems, respectively. Material heterogeneity is neglected in the macro-scale, in which the problem is defined as homogeneous continuum. On the other hand, in the fine-scale analysis, material heterogeneity is modeled by means of the discrete meso-scale model. As shown in Figure $3 \mathrm{~b}, \mathbf{X}$ is the vector that connects the origin of the global macro-scale coordinate system to the mass center of a generic RVE. In Figure 3k, an enlarged representation of the macro-scale material point is depicted in the local meso-scale coordinate system $\mathbf{y}$ as a representative volume of the heterogeneous material. It must be noted that, according to the rule of separation of scales, particles $I$ and $J$ shown in Figure $3 \mathrm{~b}$ in the macroscale local coordinate system should be illustrated mush smaller compared to their size in Figure 3 c. This is ignored for the sake of clarity. According to the rule of separation of scales, the macro- and meso-scale coordinate systems are linked as follows

$$
\mathbf{x}=\eta \mathbf{y} \quad 0<\eta<<1
$$

where $\eta$ is a very small positive scalar. To derive the governing equations that rule the problem at different length scales, asymptotic definition of displacement and rotation fields are taken into account. Therefore, the displacement and rotation of a generic node $\mathrm{P}_{I}, \mathbf{U}^{I}=\mathbf{u}\left(\mathbf{x}^{I}, \mathbf{y}^{I}\right)$ and $\Theta^{I}=\boldsymbol{\theta}\left(\mathbf{x}^{I}, \mathbf{y}^{I}\right)$, can be approximated by virtue of the following asymptotic expansions

$$
\begin{gathered}
\mathbf{u}(\mathbf{x}, \mathbf{y}) \approx \mathbf{u}^{0}(\mathbf{x}, \mathbf{y})+\eta \mathbf{u}^{1}(\mathbf{x}, \mathbf{y}) \\
\boldsymbol{\theta}(\mathbf{x}, \mathbf{y}) \approx \eta^{-1} \omega^{0}(\mathbf{x}, \mathbf{y})+\varphi^{0}(\mathbf{x}, \mathbf{y})+\omega^{1}(\mathbf{x}, \mathbf{y})+ \\
\eta \varphi^{1}(\mathbf{x}, \mathbf{y})
\end{gathered}
$$

In the above equations, terms of order $O\left(\eta^{2}\right)$ and higher are neglected. Functions $\mathbf{u}^{0}(\mathbf{x}, \mathbf{y})$, and $\mathbf{u}^{1}(\mathbf{x}, \mathbf{y})$ are continuous with respect to $\mathbf{x}$ and discrete (e.i. defined only at finite number of points) with respect to $\mathbf{y}$. It should be noted that the asymptotic expansion of rotation field is written considering the fact that, in general, rotation vector have similar meaning of the curl of displacement vector. Therefore, if one considers a two scale dependent continuous displacement-like field $\mathbf{d}^{\eta}(\mathbf{x}, \mathbf{y})$, then $2 \omega^{0}=\nabla_{y} \times \mathbf{d}^{0} ; 2 \varphi^{0}=\nabla_{x} \times \mathbf{d}^{0} ; 2 \omega^{1}=\nabla_{y} \times \mathbf{d}^{1} ;$ $2 \varphi^{1}=\nabla_{x} \times \mathbf{d}^{1}$; and subscripts $x$ and $y$ identify the nabla operator in the coarse- and finescale, respectively. Therefore, $\omega^{0}, \omega^{1}$ are the fine-scale rotations, whereas $\varphi^{0}$ and $\varphi^{1}$ are the corresponding coarse-scale rotations.

By substituting Equations 13 and 14 into the expressions of facet strains and curvatures, Equations 8 and 9, along with some mathematical manipulation, one obtains the following form for the multiple scale definition of facet strains and curvatures (check Reference [19] for all the derivation details and definition of terms of different orders)

$$
\begin{gathered}
\epsilon_{\alpha}=\eta^{-1} \epsilon_{\alpha}^{-1}+\epsilon_{\alpha}^{0}+\eta \epsilon_{\alpha}^{1} \\
\eta \chi_{\alpha}=\eta^{-1} \psi_{\alpha}^{-1}+\psi_{\alpha}^{0}+\eta \psi_{\alpha}^{1}
\end{gathered}
$$

One can formulate the multiple scale definition of facet elastic tractions as $t_{\alpha}=\eta^{-1} t_{\alpha}^{-1}+$ 


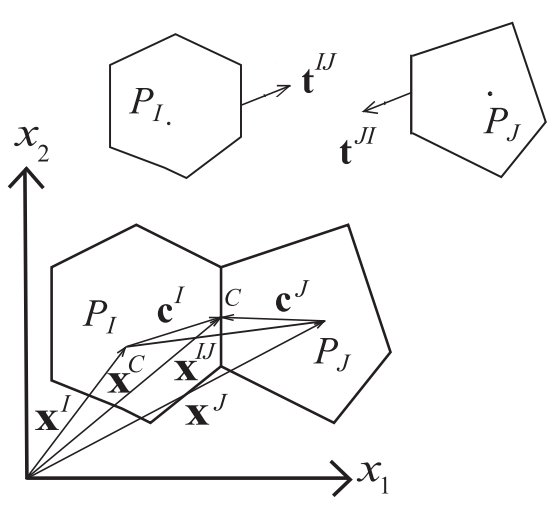

(a)

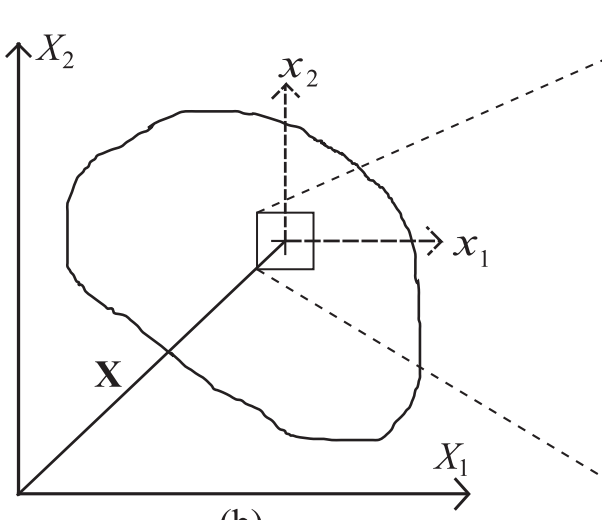

(b)

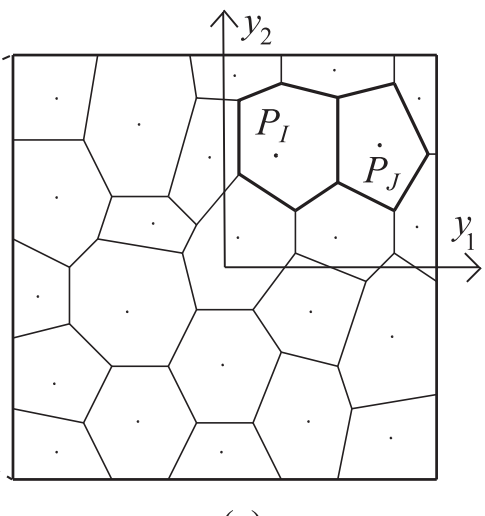

(c)

Figure 2: Geometrical explanation of the two-scale problem: (a) Geometry of two neighboring particles. (b) Macro material domain. (c) Mesoscale domain with material heterogeneity. (d) Coordinate transformations in the RVE analysis.

$t_{\alpha}^{0}+\eta t_{\alpha}^{1}$ considering $t_{\alpha}^{(\cdot)}=E_{\alpha} \epsilon_{\alpha}^{(\cdot)}$ and assuming $E_{\alpha} \sim O(1)$. It is mathematically proven that this asymptotic form holds for the case of facet nonlinear constitutive equations as well [19]. Using asymptotic expansion of facet traction in equilibrium equations and some mathematical derivation, one can derive governing equations for different scales $O\left(\eta^{-2}\right), O\left(\eta^{-1}\right)$, and $O\left(\eta^{0}\right)$. Final equations that are obtained for each scale is presented in the next sections.

\subsubsection{Fine-scale equation: the RVE prob- lem}

$O\left(\eta^{-2}\right)$ equilibrium equations refer to the rigid body motion of the RVE:

$$
u_{i}^{0}(\mathbf{X}, \mathbf{y})=v_{i}^{0}(\mathbf{X})+\varepsilon_{i j k} y_{k} \omega_{j}^{0}(\mathbf{X})
$$

One must consider that the field variables $\mathbf{v}^{0}$ and $\omega^{0}$ are only dependent on the macroscopic coordinate system $\mathbf{X}$, which implies that these quantities varies smoothly in the macro-scale material domain, while they are constant over the RVE domain. Hence, $\mathbf{v}^{0}$ and $\omega^{0}$ correspond to the macroscopic displacement and rotation fields, respectively. Considering the fact that $\omega^{0}$ represents RVE rigid rotation and taking into account the definition of $\varphi^{0}$ and $\omega^{0}$, one can conclude $\varphi^{0}=\omega^{0}$ and $\varphi^{0}$ is equal for all RVE particles.
$O\left(\eta^{-1}\right)$ governing Equations are relevant to the RVE problem, which is derived as

$$
\sum_{\mathcal{F}_{I}} A t_{\alpha}^{0} \mathbf{e}_{\alpha}^{I J}=0 ; \quad \sum_{\mathcal{F}_{I}} A\left(\mathbf{c}^{I} \times t_{\alpha}^{0} \mathbf{e}_{\alpha}^{I J}\right)=0
$$

Equation 18 is the force and moment equilibrium equations of every single particle inside the RVE subjected to $O(1)$ facet traction $t_{\alpha}^{0}$ vector, which, in turn, is a function of $\epsilon_{\alpha}^{0}$ that is written as

$$
\begin{aligned}
\epsilon_{\alpha}^{0} & =r^{-1}\left(u_{i}^{1 J}-u_{i}^{1 I}+\varepsilon_{i j k} \omega_{j}^{1 J} c_{k}^{J}-\varepsilon_{i j k} \omega_{j}^{1 I} c_{k}^{I}\right) e_{\alpha i}^{I J} \\
& +P_{i j}^{\alpha}\left(\gamma_{i j}+\varepsilon_{j m n} \kappa_{i m} y_{n}^{c}\right)
\end{aligned}
$$

where $\gamma_{i j}=v_{j, i}^{0}-\varepsilon_{i j k} \omega_{k}^{0}, \quad \kappa_{i j}=\omega_{j, i}^{0}$ are the macro-scale Cosserat strain and curvature tensors, respectively. The vector $\mathbf{y}^{c}$ is the position vector of the facet centroid shared between particles $I$ and $J$ in the local lower-scale coordinate system, shown in Figure 3 a. $P_{i j}^{\alpha}=n_{i}^{I J} e_{\alpha j}^{I J}$ is an operator to project macroscopic strain and curvature tensors onto the RVE facets as normal or tangential strain components. Considering Equation 4, it is concluded that the first term in Equation 19 is the fine-scale definition of the facet normal and tangential strains written in terms of lower-scale displacement and rotation fields $u^{1}$ and $\omega^{1}$. Furthermore, the second term in Equation 19, $P_{i j}^{\alpha}\left(\gamma_{i j}+\varepsilon_{j m n} \kappa_{i m} y_{n}^{c}\right)$, is the projection of the macroscale Cosserat strain and 
curvature tensors for each RVE facet. Therefore, Equation 19 states that the $O(1)$ strains on each RVE facet is the sum of their fine-scale strains and the projection of the macroscopic strain tensor onto that facet.

\subsubsection{Coarse-scale equation: the macro- scopic problem}

Mathematical manipulation of the $O(1)$ terms in the multiple-scale governing equations leads to the macroscopic translational and rotational equilibrium equations. The following equations are derived for the coarse-scale equilibrium equations and their corresponding homogenized stress and moment stress tensors

$$
\begin{gathered}
\sigma_{j i, j}^{0}+b_{i}=0 \\
\sigma_{i j}^{0}=\frac{1}{2 V_{0}} \sum_{I} \sum_{\mathcal{F}_{I}} A r t_{\alpha}^{0} P_{i j}^{\alpha}
\end{gathered}
$$

and

$$
\begin{gathered}
\varepsilon_{i j k} \sigma_{i j}^{0}+\frac{\partial \mu_{j i}^{0}}{\partial x_{j}}=0 \\
\mu_{i j}^{0}=\frac{1}{2 V_{0}} \sum_{I} \sum_{\mathcal{F}_{I}} A r t_{\alpha}^{0} Q_{i j}^{\alpha}
\end{gathered}
$$

where $V_{0}$ is the volume of the RVE; $\rho_{u}=$ $\sum_{I} M_{u}^{I} / V_{0}$ is the mass density of the macroscopic continuum. Equation 20 corresponds to the classical differential form of equilibrium equation. In addition, Equation 21 defines macroscopic stress tensor in terms of the solution of the RVE problem. Matrix $Q_{i j}^{\alpha}$ is defined as $Q_{i j}^{\alpha}=n_{i}^{I J} \varepsilon_{j k l} x_{k}^{C} e_{\alpha l}^{I J}$. $\mu_{i j}^{0}$ is the macroscopic moment stress tensor calculated using the results of RVE analysis, and Equation 22 corresponds to the classical rotational equilibrium equation of Cosserat continua. It is shown numerically that the anti-symmetric part of the RVE homogenized stress tensor tends to be negligible for different types of loading condition [19]. Therefore, it is rational to neglect the moment stress tensor effect in the numerical examples in the next section and only consider the response in terms of the symmetric stress tensor.

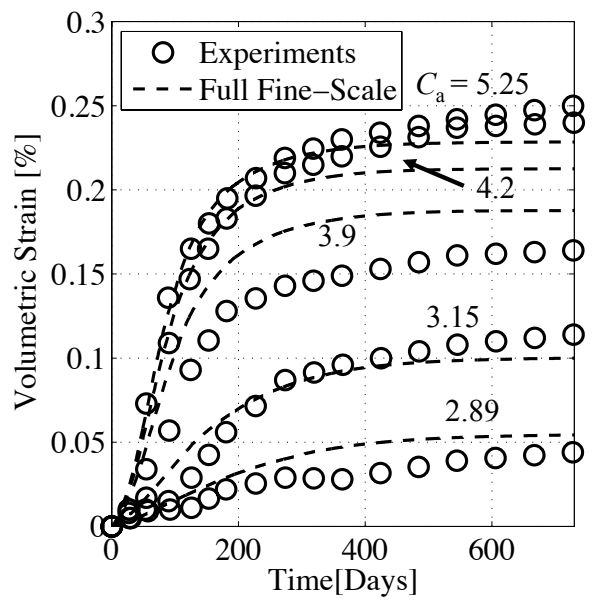

Figure 4: Volumetric expansion of concrete prism for different alkali contents by experiment and full fine-scale analysis.

\section{Numerical results}

In this section, effect of ASR expansion and different values of alkali content are investigated for multiple concrete prisms that were studied experimentally [23], by means of both full fine-scale analysis and the two-scale homogenization framework presented in this paper. Free expansion of sealed concrete prisms of dimension $75 \mathrm{~mm} \times 75 \mathrm{~mm} \times 265 \mathrm{~mm}$ are recorded for 320 days. Aggregate size distribution of the concrete used in experiments is: $1 / 3$ of aggregate particles between 4.75 and 9.5 $\mathrm{mm}, 1 / 3$ between 9.5 and $12.5 \mathrm{~mm}$ and the last third between 12.5 and $19.0 \mathrm{~mm}$. For the generation of prisms that are completely modeled with LDPM for full-fine scale analysis in addition to the LDPM RVEs used in multiscale homogenization study, the following parameters are used: minimum aggregate size, $d_{0}=4.75$ $\mathrm{mm}$; maximum aggregate size, $d_{a}=19 \mathrm{~mm}$; fuller curve exponent, $n_{F}=0.55$; and the following mix composition: cement content, $c=$ $420 \mathrm{~kg} / \mathrm{m}^{3}$; water to cement ratio, $w / c=0.45$; aggregate to cement ratio, $a / c=4.25$. Figure 3 shows the geometrical configuration simulated for the two separate analysis: Figure 3(b) shows the polyhedral cell representation of a prism that is fully generated by LDPM, while Figure 3(a) presents the homogenization model in which the prism is discretized by three hexahe- 


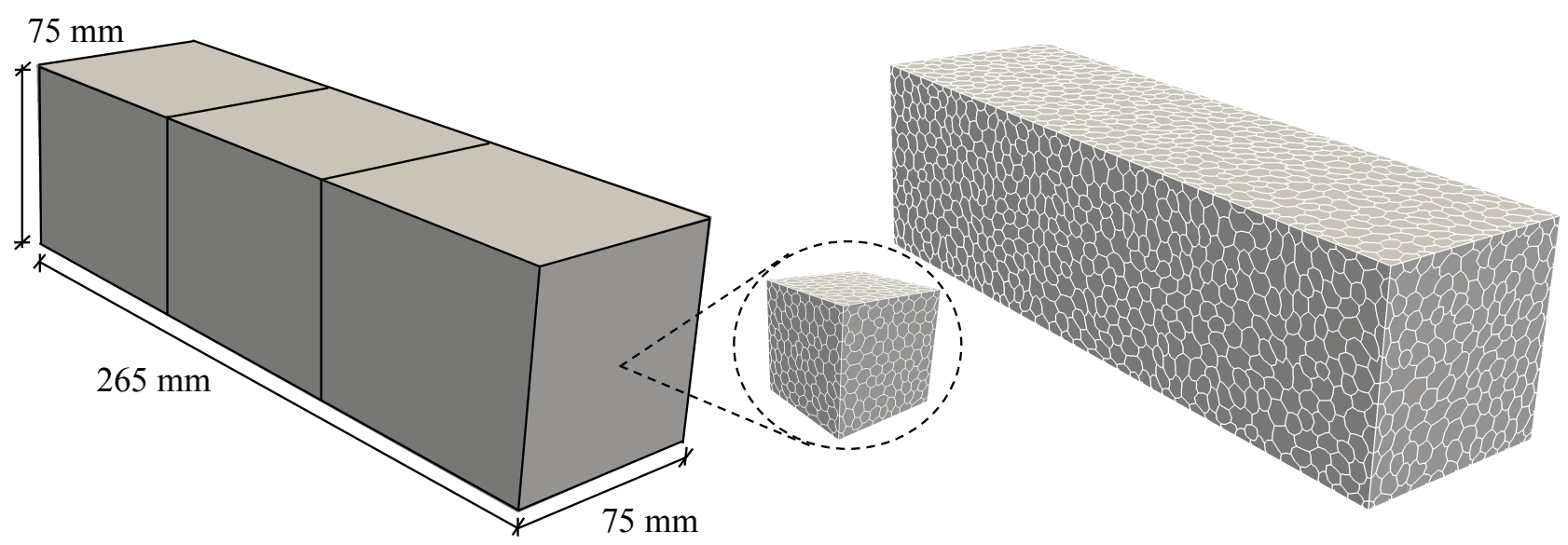

Figure 3: (Left) Two-scale homogenization problem. (Right) Full mesoscale prism model.

dral finite elements with one integration point. A single RVE created by LDPM is assigned to each integration point to calculate the effective material response during the analysis, see Figure $3(a)$.

Five levels of alkali content: $c_{a}=2.89,3.15$, $3.9,4.2$, and $5.25 \mathrm{~kg} / \mathrm{m}^{3}$ are considered to investigate its effect on ASR analysis. For each alkali content, full fine-scale analysis is carried out for three different LDPM mesh realizations to examine the aggregate distribution effect, and the average response is presented. Evolution of volumetric strain versus time of the full finescale analysis and corresponding experimental data are plotted in Figure 4, One can see that the computational results match well with the experimental data. One can see that the rate of volumetric strain decreases and plateaus as ASR reaction progresses. The ASR parameters are first calibrated for the $2.89 \mathrm{~kg} / \mathrm{m}^{3}$ alkali content and then used to validate the response for other alkali content values. Calibrated parameters are: $k_{a}=1, C_{a}^{0}=2.7 \mathrm{~kg} / \mathrm{m}^{3}, C_{a}^{1}=$ $4.37 \mathrm{~kg} / \mathrm{m}^{3}, a_{s 0}=3.45 \mathrm{e}-13 \mathrm{~m} \times \mathrm{m} / \mathrm{sec}, C_{i}^{1}=$ $28 \mathrm{e}-11 \mathrm{~m} \times \mathrm{m} / \mathrm{sec}, \eta=439,540 \mathrm{~kg}^{-1}, k_{g}=689$ $\mathrm{kg} / \mathrm{m}^{3}, E_{a g}=E_{a d}=500 \mathrm{~J} / \mathrm{mol}$. Current $T$ and reference temperature $T_{0}$ are considered to be equal which means temperature effects are neglected. It should be noted that the specimens are sealed which maintains the relative humidity as high values very close to $100 \%$. LDPM parameters used in the simulation are: $E_{N}=60$ $\mathrm{GPa}, \sigma_{t}=4.75 \mathrm{MPa}, \sigma_{c 0}=150 \mathrm{MPa}, \alpha=0.25$, $n_{t}=0.2, l_{t}=75 \mathrm{~mm}, r_{s t}=2.6, H_{c 0} / E_{0}=0.4$, $\mu_{0}=0.4, \mu_{\infty}=0, k_{c 1}=1, k_{c 2}=5, \sigma_{N 0}=600$ $\mathrm{MPa}, \alpha=E_{T} / E_{N}=0.25$.

The same LDPM and ASR model parameters are used in the homogenization analysis. To investigate particle distribution and RVE size effect, three different RVE sizes: $35,50,75 \mathrm{~mm}$ are considered, and six different mesh realizations are examined for each size. The overall multiscale numerical procedure adopted in this paper can be summarized as follows.

- The finite element method is employed to solve the macro-scale homogeneous problem in which external loads and essential BCs are applied incrementally. During each numerical step, strain increments $\Delta \gamma_{i j}=\Delta v_{j, i}^{0}-\varepsilon_{i j k} \Delta \varphi_{k}^{0}$ and curvature increments $\Delta \kappa_{i j}=\Delta \omega_{j, i}^{0}$ tensors are calculated at each integration point based on the nodal displacement and rotation increments of the corresponding finite element.

- Increment of ASR induced normal strain $\Delta \epsilon_{N}^{a s r}$, as discussed in Section 2.3, are calculated for all facets inside RVE.

- The macroscopic strain and curvature increments are projected onto the RVE facets through the proper projection operators: $\Delta \epsilon_{\alpha}^{c}=P_{i j}^{\alpha}\left(\Delta \gamma_{i j}+\varepsilon_{j m n} \Delta \kappa_{i m} y_{n}^{c}\right)$. These projected strains along with ASR induced normal strain are imposed, upon 


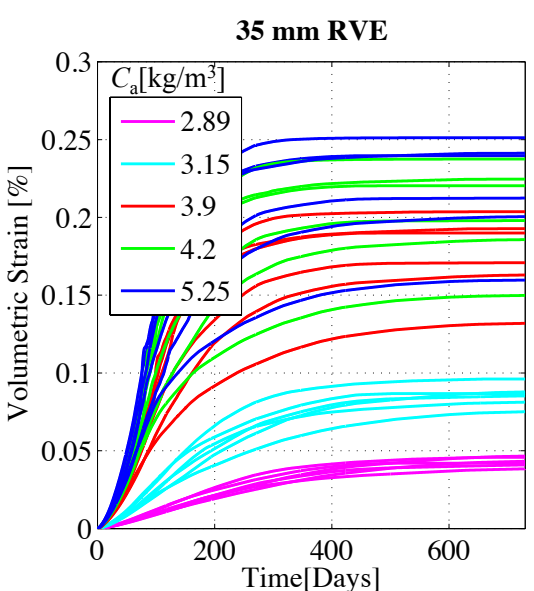

(a)

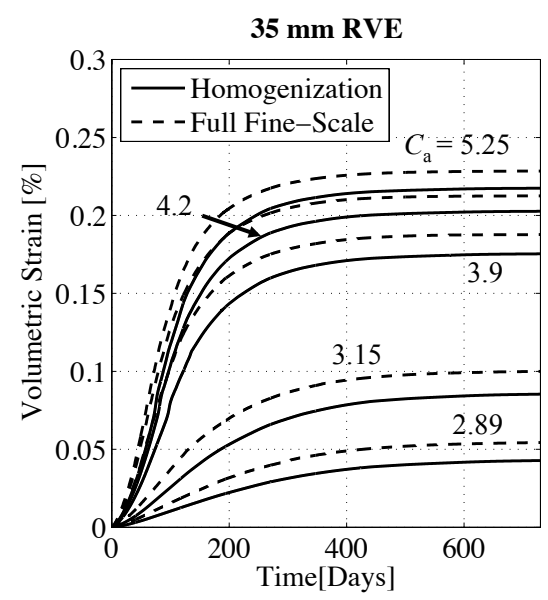

(d)

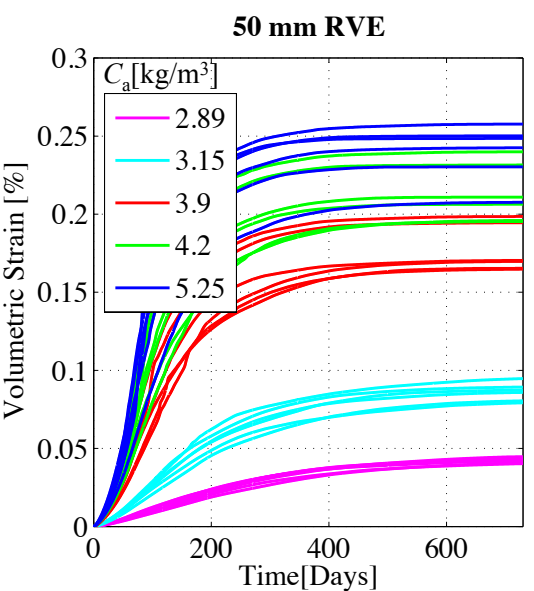

(b)

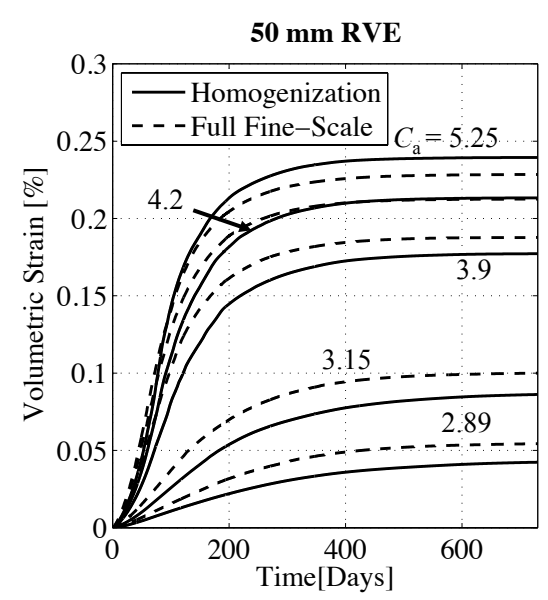

(e)

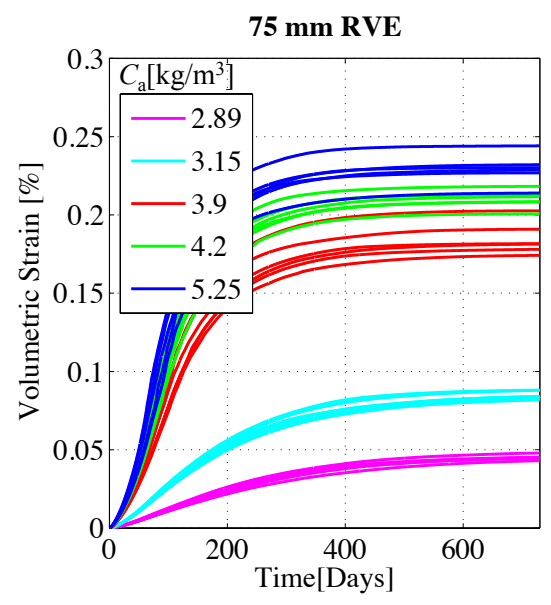

(c)

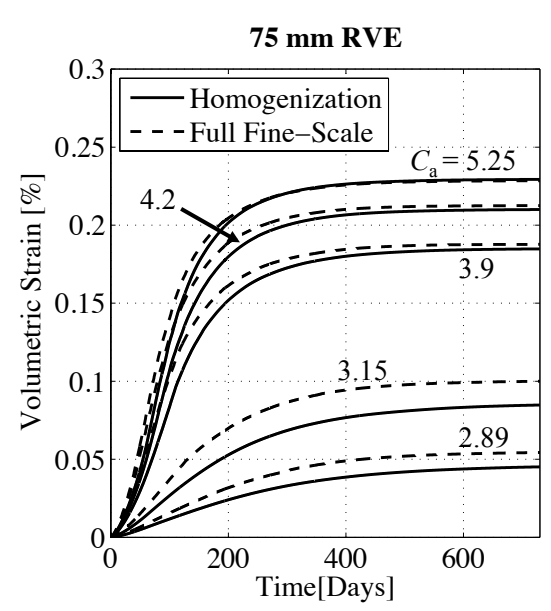

(f)

Figure 5: Volumetric expansion of the prisms with different alkali contents obtained from homogenization framework using RVE size of (a) $35 \mathrm{~mm}$ (b) $50 \mathrm{~mm}$, and (c) $75 \mathrm{~mm}$. (d) to (f) Comparison of the homogenization results using different RVE sizes with the full fine-scale ones.

sign change shown in the following equation, as eigen-strains to the RVE allowing the calculation of the fine-scale solution governed by the fine-scale constitutive equations.

$$
\begin{gathered}
\Delta \epsilon_{\alpha}^{0}=\Delta \epsilon_{\alpha}^{f}+\Delta \epsilon_{\alpha}^{c}-\Delta \epsilon_{N}^{a s r} \delta_{\alpha N} \\
=\Delta \epsilon_{\alpha}^{f}-\left(-\Delta \epsilon_{\alpha}^{c}\right)-\Delta \epsilon_{N}^{a s r} \delta_{\alpha N}
\end{gathered}
$$

$\delta_{\alpha N}$ is 1 for $\alpha=N$ and 0 for $\alpha=M$ or $L$.

- Finally, the fine-scale facet tractions are used to compute, through Equations 21. the macroscopic stresses for each Gauss point in the FE mesh.
Variation of volumetric expansion versus time is plotted for different RVE sizes in Figures 5(a) to (c). Each plot consist of six different curves corresponding to different particle distribution for each level of alkali content. For each value of alkali content, one can see that the curves are more scattered for $35 \mathrm{~mm}$ RVE than $50 \mathrm{~mm}$ and for $50 \mathrm{~mm}$ compared to $75 \mathrm{~mm}$ RVE size. Therefore, it is concluded that higher ratio of the RVE size to the maximum particle size results in smaller standard deviation in the response. In addition, since higher value of alkali content leads to higher level of ASR damage and subsequently enhanced inhomogeneity of the damaged RVE, one can observe that, for 
each RVE size, the curves are more scattered for higher level of alkali content. There results are averaged for each RVE size and different alkali contents and compared to the full fine-scale analysis results in Figures 5(d) to (f). One can see that the homogenization and the full finescale results match well for different RVE sizes and alkali content values. The homogenization results agrees more with the full fine-scale simulations as the RVE size is increased. This is expected since larger RVEs are better representations of lower-scale material structure.

Finally, the significant capability of the developed homogenization framework is enormous saving of computational cost, while it retains adequate precision of the results. Homogenization analysis of the prism using 75 mm RVE approximately takes 6 hours which is close to full fine-scale analysis. But, analyses with $50 \mathrm{~mm}$ and $35 \mathrm{~mm}$ RVEs take 1.5 hours and 40 minute, respectively. It should be noted that only three finite elements are used in the homogenization analysis. Therefore, computational time saving in the analysis of larger structures discretized by large number of finite elements will be more considerable. This is the main purpose of developing a multiscale framework which is successfully accomplished by means of the developed homogenization scheme.

\section{Conclusions}

A multiscale homogenization framework for the simulation ASR effect on concrete is presented. Considerable save of the computational cost is accomplished while the results are correlated well with the experimental data. RVE size to maximum aggregate size ratio is the key factor in the scatter of the results, which decreases as this ratio is increased. It is shown that the homogenization framework replicates the full fine-scale analysis results with high accuracy using RVEs smaller than the volume associated with the related Gauss point in the finite element model. ASR degradation effect is a proper application to use homogenization framework, since the related damage pattern is spread throughout the specimen rather than localized form which appears in fracture problems.

\section{REFERENCES}

[1] T. E. Stanton, Expansion of concrete through reaction between cement and aggregate, Proceeding American Society of Civil Engineers, 66:1781-1811, 1940.

[2] Z. P. Bazănt, G. Zi, and C. Meyer, Fracture mechanics of ASR in concretes with waste glass particles of different sizes, Journal of Engineering Mechanics, 126(3):226-232, 2000.

[3] R. G. Charlwood, S. V. Solymar, and D. D. Curtis, A review of alkali aggregate reactions in hydroelectric plants and dams, in Proceedings of the International Conference of Alkali-Aggregate Reactions in Hydroelectric Plants and Dams, volume 129, Fredericton, Canada, 1992.

[4] E. M. R. Fairbairn, F. L. B. Ribeiro, L. E. Lopes, R. D. Toledo-Filho, and M. M. Silvoso, Modelling the structural behaviour of a dam affected by alkali-silica reaction, Communications in Numerical Methods in Engineering, 22(1):1-12, 2005.

[5] F. Ulm, O. Coussy, L. Kefei, and C. Larive, Thermo-Chemo-Mechanics Of ASR Expansion In Concrete Structures, Journal of Engineering Mechanics, 126(3):233-242, 2000.

[6] V. Saouma and L. Perotti, Constitutive model for alkali-aggregate reactions, $A C I$ Materials Journal, 103:194—202, 2006.

[7] C. Comi, R. Fedele, and U. Perego, A chemo-thermo-damage model for the analysis of concrete dams affected by alkali-silica reaction, Mechanics of Materials, 41(3):210-230, 2009.

[8] S. Poyet, A. Sellier, B. Capra, G. Foray, J. M. Torrenti, H. Cognon, and E. Bour- 
darot, Chemical modelling of alkali silica reaction: Influence of the reactive aggregate size distribution, Materials and Structures, 40(2):229-239, 2007.

[9] B. Capra and A. Sellier, Orthotropic modelling of alkali-aggregate reaction in concrete structures: numerical simulations, Mechanics of Materials Journal, 35:817830, 2003.

[10] V. E. Saouma, R. A. Martin, M. A. HaririArdebili, T. Katayama. A mathematical model for the kinetics of the alkaliâĂŞsilica chemical reaction. Cement and Concrete Research 2015; 68: 184-âĂŞ195.

[11] G. Cusatis, R. Rezakhani, M. Alnaggar, $\mathrm{X}$. Zhou, and D. Pelessone, Multiscale computational models for the simulation of concrete materials and structures, pages 23-38, CRC Press, 2015/02/26 2014, ISBN 978-1-138-00145-9.

[12] M. H. Motamedi, C. D. Foster. An improved implicit numerical integration of a non-associated, three-invariant cap plasticity model with mixed isotropickinematic hardening for geomaterials. International Journal for Numerical and Analytical Methods in Geomechanics 2015; 39: 1853-1883.

[13] M. Alnaggar, G. Cusatis, and G. DiLuzio, Lattice Discrete Particle Modeling (LDPM) of Alkali Silica Reaction (ASR) deterioration of concrete structures, $\mathrm{Ce}$ ment and Concrete Composites, 41:45-59, 2013. doi:10.1201/b16645-5.

[14] M. Alnaggar, M. Liu, J. Qu . G. Cusatis. Lattice Discrete Particle Modeling of acoustic nonlinearity change in accelerated alkali silica reaction (ASR) tests. Materials and Structures 2015; DOI 10.1617/s11527-015-0737-9.

[15] G. Cusatis, D. Pelessone, A. Mencarelli. Lattice Discrete Particle Model (LDPM) for failure behavior of concrete. I: Theory. Cement and Concrete Composites 2011; 33: 881-890.
[16] G. Cusatis, A. Mencarelli, D. Pelessone, J. Baylot. Lattice Discrete Particle Model (LDPM) for failure behavior of concrete. II: Calibration and validation. Cement and Concrete Composites 2011; 33: 891-905.

[17] F. J. Vernerey and M. Kabiri. Adaptive Concurrent Multiscale model for fracture and crack propagation in heterogeneous material. Comput. Methods Appl. Mech. Engrg. 2014; 276: 566-588.

[18] T. Wu, I. Temizer, P. Wriggers. Multiscale hydro-thermo-chemo-mechanical coupling: Application to alkali-silica reaction. Computational Materials Science 2014, 84: 381-âĂŞ395.

[19] R. Rezakhani, G. Cusatis, Asymptotic expansion homogenization of discrete finescale models with rotational degrees of freedom for the simulation of quasi-brittle materials. J. Mech. Phys. Solids 2016; 88: 320-345.

[20] R. Rezakhani and G. Cusatis, Generalized mathematical homogenization of the lattice discrete particle model, in Proceedings of the 8th International Conference on Fracture Mechanics of Concrete and Concrete Structures, FraMCoS 2013, pages 261-271, Toledo, Spain, 2013.

[21] G. Cusatis, M. Alnaggar, R. Rezakhani. Multiscale modeling of Alkali Silica reaction degradation of concrete. CONMOD 2014: 431-438, DOI: 10.13140/2.1.3783.4561.

[22] MARS: Modeling and Analysis of the Response of Structures - User's Manual, D. Pelessone, ES3, Solana Beach (CA), USA. http://www.es3inc.com/mechanics/ MARS/Online/MarsManual.htm, 2009.

[23] M.H. Shehata, M.D. Thomas. The effect of fly ash composition on the expansion of concrete due to alkali-silica reaction. Cem Concr Res 2000; 30: 1063-72. 